\title{
Image enhancement technology research for army applications
}

\author{
Piet B.W. Schwering", Rob A.W. Kemp, Klamer Schutte \\ TNO, PO Box 96864, 2509 JG The Hague, The Netherlands;
}

\begin{abstract}
Recognition and identification ranges are limited to the quality of the images. Both the received contrast and the spatial resolution determine if objects are recognizable. Several aspects affect the image quality. First of all the sensor itself. The image quality depends on the size of the infrared detector array and the sensitivity. Second, also the intervening atmosphere, in particular over longer ranges, has an impact on the image quality. It degrades the contrast, due to transmission effects, as well as it influences the resolution, due to turbulence blur, of the image. We present studies in the field of infrared image enhancement. Several techniques are described: noise reduction, super resolution, turbulence compensation, contrast enhancement, stabilization. These techniques operate in real-time on COTS/MOTS platforms. They are especially effective in the army theatre, where long horizontal paths, and short line-of-sight limited urban operations are both present. Application of these techniques on observation masts, such as on military camp sites, and on UAVs and moving ground vehicles are discussed. Examples will be presented from several trials in which these techniques were demonstrated, including the presentation of test results.
\end{abstract}

Keywords: Image enhancement, super resolution, noise reduction, turbulence compensation, quantitative evaluation

\section{INTRODUCTION}

In the army environment several techniques are being studied for the improvement of situational awareness. At the starting point are techniques that provide improvement of the infrared image quality, such as resolution enhancement and noise reduction. With this information it is easier for the operator to make faster and more correct decisions. These technologies are applicable to, in particular, army applications. Application is foreseen in moving vehicles as well as stationary platforms as masts. Beside technical descriptions, examples and test results will be presented.

In army scenarios situational awareness is of great importance. In general, techniques are studied for general improvements on situational awareness, enhancing the image quality as noise reduction, resolution enhancement and contrast enhancement. This will allow for a better separation between foreground and its background - in many modern army scenarios we need to discern objects of interest from a fairly similar but benign background. In long range applications over land, turbulence effects play an important role. These effects are the limiting factor of the image resolution of the sensor. Compensation for turbulence, in combination with long range image stabilization, is therefore an important topic to study. In short range applications, such as operations in urban environments, short reaction times and a large field of regard are important. The nature of threats are very different in these environments, and lead to requirements on automated processing, and combination of different sensor field of views. Techniques as image stitching, and recognition of threat and their behavior are important in these environments. When provided with higher quality images and robust target data that increases the confidence of the image data, the operator will be able to perform his task longer. The requirements for fast reaction times in short range application require that technologies are applied in real-time applications. With the application of the image enhancement technologies to the same sensors additional detection, recognition and identification capability is released in these army scenarios.

\footnotetext{
* piet.schwering@tno.nl; phone +31 8886641 10; http://www.tno.nl/
} 
In this paper we analyze the status of the different techniques for operational applications. In section 2 we discuss the rational of image enhancement technology and the way to obtain operational systems. Different image enhancement technologies are descried in section 3. In section 4 we discuss performance assessment, and in section 5 we present conclusions.

\section{RATIONALE FOR IMAGE ENHANCEMENT TECHNOLOGY}

\subsection{Rationale}

Image enhancement technology can provide the means to overcome the limitations set by sensor and environment, that are presented in the introduction. Of importance is the fact that the operator can perform the task better. Normally these tasks are defined as detection, recognition and identification of persons, objects and targets. This means that this operator should have tools that enable him to have improved vision. Improved task performance can be described in terms of

- Longer range vision

- Faster observation

- More comfortable observation

These terms address the factors in the introduction that are relevant for long range and short range observation: Improving reaction time, longer range capability, and less stress in observing large amounts of high quality data. With enhanced imagery the operator focus longer and perform his task with higher confidence.

\subsection{Operational support with image enhancement}

To obtain deployment and implementation of the techniques, an overall design must be provided in which the different techniques can be applied. For imaging sensors that provide both automated detections and image results after enhancement, a schedule was designed in Schwering et al. (2007)[1]. This schedule is given in Figure 1. There are different blocks for signal conditioning, which provides for image enhancement, and for automated detection and classification.

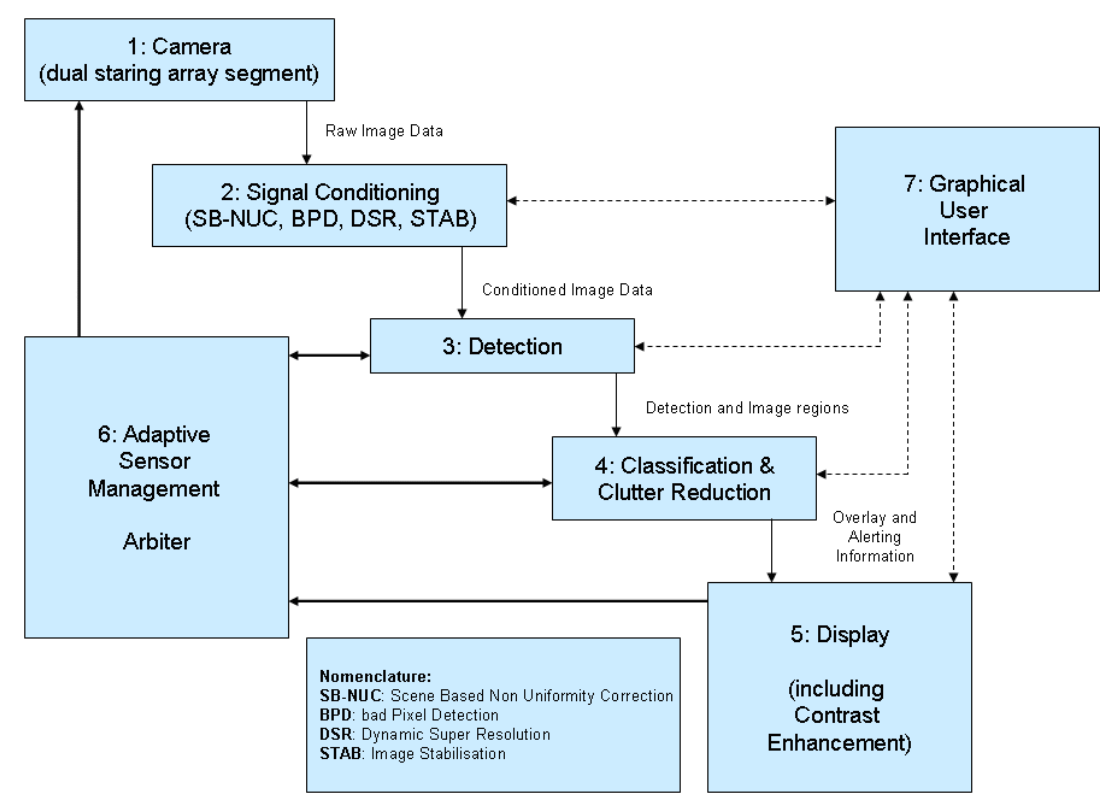

Figure 1. Block diagram example for the implementation of the image enhancement tools, together with automated detection and classification 
Each application requires a different block diagram design. In the approach above we seek automated detections on the already processed imagery, making use of the enhanced image quality. For the detection of persons or small objects separate detection (without image enhancement but with turbulence mitigation) and classification (with image enhancement) are more suitable, as there is no information on the source blob itself. This process can be managed by the adaptive sensor management.

In Table 1 below we list an overview of the image enhancement techniques. The list gives a description of the approach and the expected results during operations.

Table 1: Overview of image enhancement techniques

\begin{tabular}{|l|l|l|}
\hline Technique & Approach & Results \\
\hline Artifact reduction & $\begin{array}{l}\text { Scene based non uniformity reduction and bad pixel } \\
\text { removal }\end{array}$ & Comfortable observation \\
\hline Contrast enhancement & Optimum display of camera image & Improve recognition \\
\hline Dynamic super resolution & More image details from camera motion & Improve recognition \\
\hline Stabilization & Images are stabilized on image content & Comfortable observation \\
\hline Stitching & Combining individual images & Situation overview \\
\hline Turbulence compensation & Compensate turbulence blur and beam wander & Improve recognition \\
\hline Automated detection & Generated target cues automatically & Information on targets \\
\hline
\end{tabular}

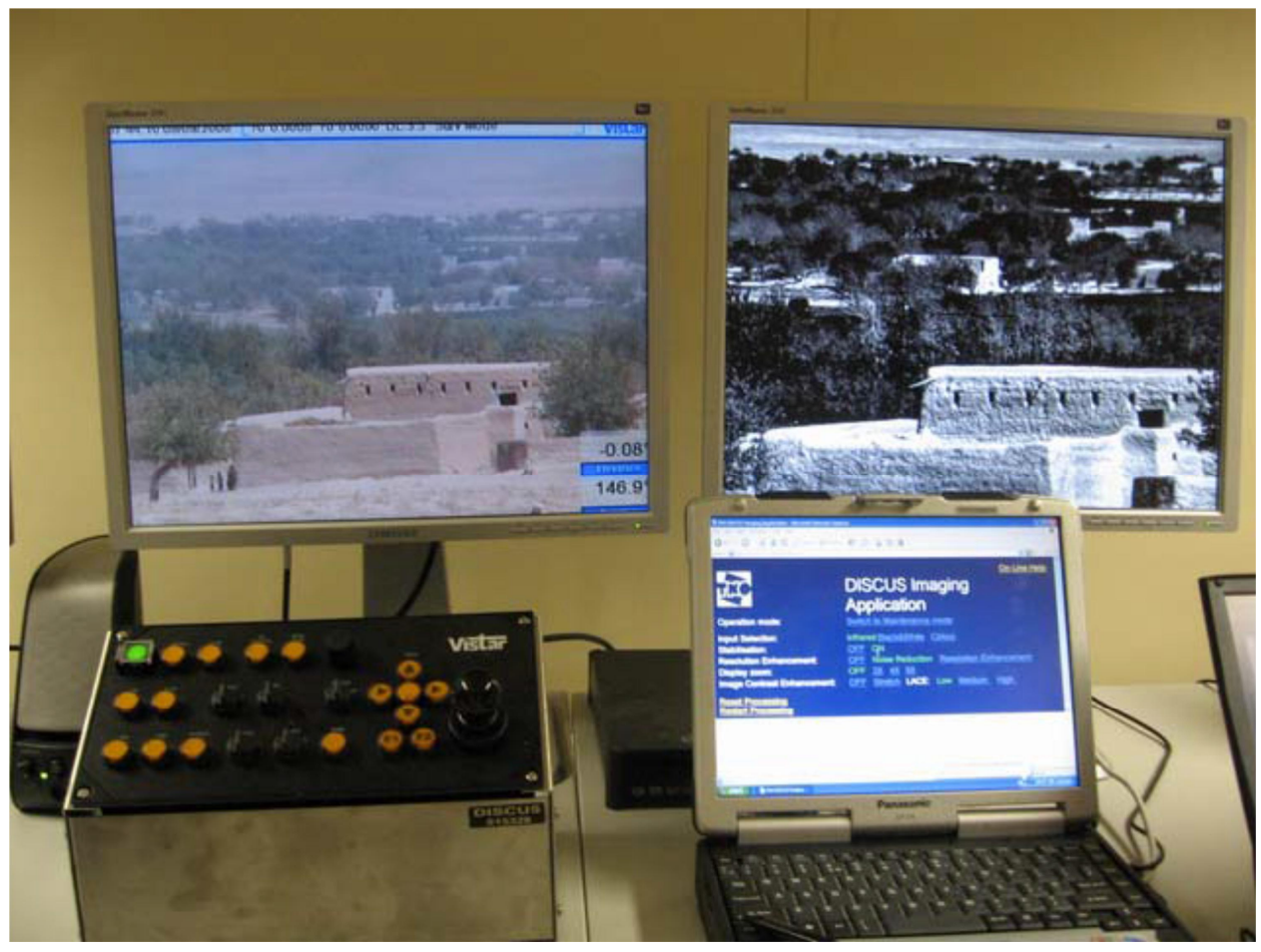

Figure 2. TNO Image Enhancement System during operation in Uruzgan, Afghanistan DISCUS (left visible light, right midwave infrared) 
Our developments have resulted in real-time hardware implementations of algorithms for scene-based non-uniformity correction, stabilization, contrast enhancement, resolution enhancement and noise reduction. These techniques, that provide an improved image, are the pre-processing stage before the detection and classification process. In the recent past the real-time image enhancement system has been demonstrated on thermal and daylight imagery in operational army environments (compound security) in Afghanistan, airborne UAV (the Sperwer Remotely Piloted Vehicle Image Enhancement) applications, and in maritime environments on-board the Netherlands air-defence and command frigates. The system is improving the situational awareness in these diverse defense operations. In Figure 2 we show the system set-up in Afghanistan where the processing is applied to imagery.

\section{IMAGE ENHANCEMENT TECHNOLOGIES}

\subsection{Local Adaptive Contrast Enhancement (LACE)}

The application of an advanced signal conditioning results in high dynamic range imagery with good signal to noise ratio. The dynamic range of such imagery can exceed the display capabilities of standard display devices, leading to a loss of information in the visualization process. To allow visualization of all information present in the high-quality imagery we propose the use of Local Adaptive Contrast Enhancement (LACE). LACE is an algorithm to compress the overall dynamic range of the images while retaining small amplitude details and maintaining an overall natural look. In addition, recent developments on LACE now also allow it to reduce noise contents based on spatial characteristics for bad signal to noise scenarios.

Local Adaptive Contrast Enhancement (LACE, Schutte et al. 2003 [2] and Dijk et al. 2007 [3]) is used only for displaying. It is effective way to map the whole dynamic range of the camera (typical 8-14 bits) on a display, while visualizing as much details as possible LACE will allow seeing details in light and dark areas without the need for dedicated gain and offsetting controls. LACE requires two input parameters, the input noise and the level of enhancement. The noise level in the input image can be supplied by the Noise Reduction algorithm. The level of enhancement depends on the task of the operator. For Situational Awareness a mild version of LACE is needed. For this task the large details are more important than the small scale details. For a detection task a medium version of LACE is needed, which enhances more small details; at the risk of reduce the general scene understanding. Mild LACE will result in a natural image, while medium LACE will be less natural with lots of details.

In Figure 3 we present an example of the application of a mild version of LACE in a harbor environment. These experiments were performed with a staring IRST making using of uncooled thermal cameras (Kemp et al, 2008 [4]) in the Rotterdam harbor. The results show clear improvement of the contrast in the scenes.
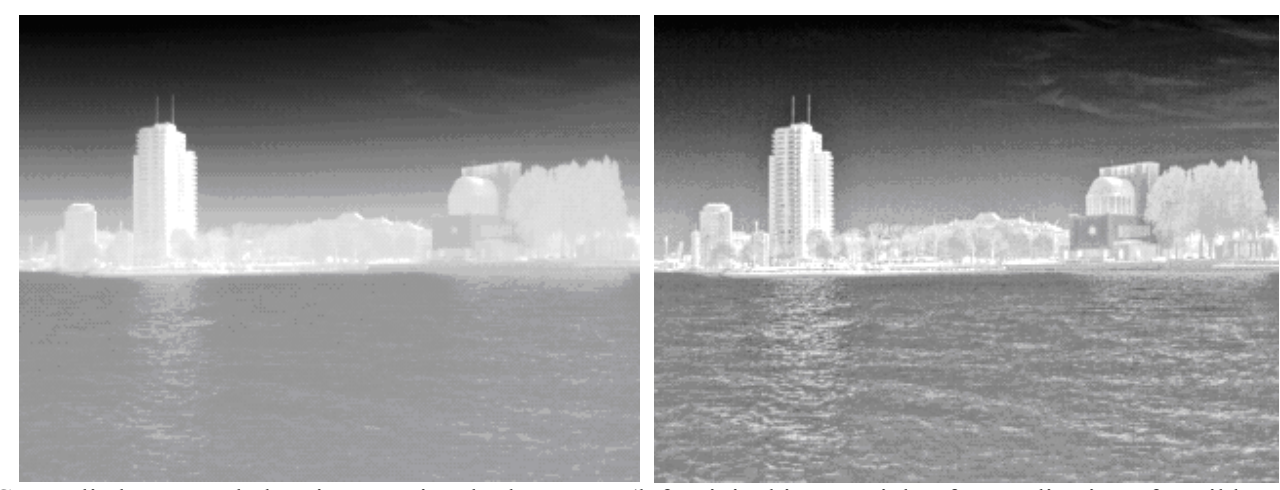

Figure 3. LACE applied to uncooled IR imagery in a harbor scene (left original image, right after application of a mild version of LACE) 


\subsection{Dynamic Super Resolution (DSR)}

The term super resolution as used in this paper describes the generation of higher resolution imagery using resolution enhancement and image sharpening techniques. The basic mechanism behind resolution enhancement (Schutte et al. 2003 [2]) is that temporal resolution of image sequences is exchanged for spatial resolution while improving the signalto-noise ratio. This means that noise reduction and resolution enhancement are combined into a single algorithm. In dynamic super resolution (DSR) several recordings of the same scene are combined to a high-resolution Image. The differences between the recordings are used to calculate a new, improved, high-resolution image. For the algorithm to be effective, the low resolution imagery needs to be registered with sub-pixel precision. Figure 4 gives a visualization of the mechanism behind DSR. The algorithm will increase the resolution by a factor of typically $2-4$. This means that for a resolution enhancement factor of 2 , there will be $2 \times 2=4$ high-resolution pixels for each low-resolution camera pixel.

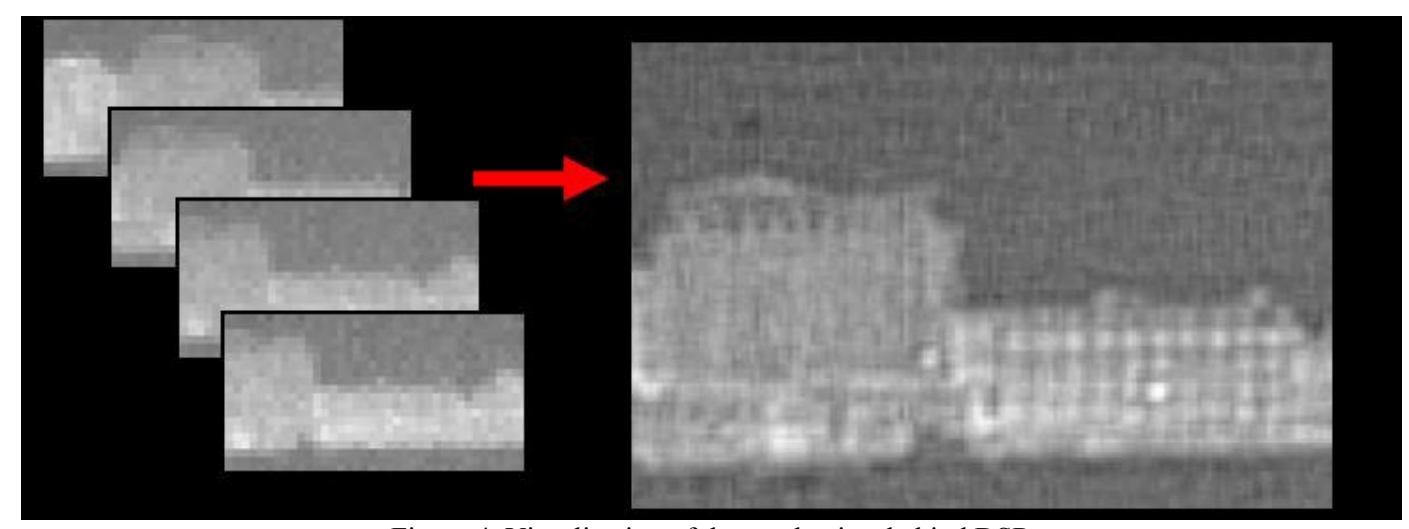

Figure 4. Visualization of the mechanism behind DSR

Since a super resolved image may even contain more detail than the input imagery, we also applied DSR4 to the input sequences. Results of the application of dynamic super resolution with a factor 4 (DSR4) are shown in Figure 5. For comparison, a region of the input image (image 180 from the sequence) has been magnified to the same size as the DSR image and a mild version of LACE was applied. Being acquired with a camera of 320x240 pixels, the image in Figure 5 looks similar to an image, acquired with a camera of 1280x960 pixels. A remarkable fact is that in the super resolved image details, hardly visible in the original image, become clearly visible in the super resolved image, thus achieving a cost effective zoom. Because of this, target identification can be done faster. The examples are from the same harbor dataset as Figure 3.
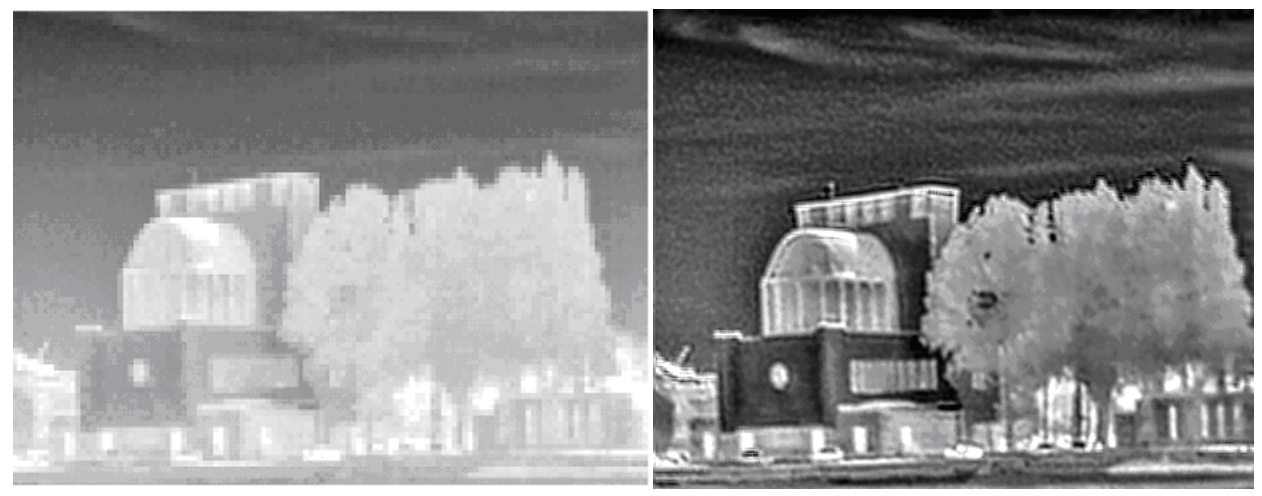

Figure 5. Applying DSR4 and LACE to uncooled IR imagery (left original image with mild LACE applied, right processed image with DSR4 with medium LACE applied) 
With megapixel cameras available for relatively low prices, as currently available in the visual band, it makes sense to replace the super resolution processing by a temporal noise reduction. DSR2 and DSR4 processing as discussed above both increase the spatial resolution as well as the signal to noise reduction. With typical megapixel CCD or CMOS sensors we propose to perform only the noise reduction instead of full DSR processing. Stabilization and turbulence mitigation can still be applied.

\subsection{Stabilization and motion estimation}

Super resolution algorithms rely on an accurate motion estimation input. This is provided by a Scene Based Motion Estimation (SBME) algorithm (Schutte et al. 2003 [2]). The advantage of an accurate SBME algorithm is that there is no need for (expensive) external motion sensors. Since the image information itself is used for motion estimation, application of an SBME algorithm eliminates the possibility of errors due to vibration between the motion sensor and the imaging system. To achieve an accurate SBME algorithm its motion model should match the actual scene motion. Currently implemented models include translation-only, affine and grid based motion models, which accurately can describe apparent scene motion caused by translations and rotations of the camera and remote scenes. Since this accurate motion estimation already is performed, other techniques such as electronic stabilization and mosaic generation can be implemented without much problems. As a matter of fact, the standard output of DSR is already stabilized, and mosaic generation is available as a standard option. Note that for some army applications including driver assistance applications a translation only or affine motion model will not be sufficient: due to the 3D nature of a scene and significant motion of the sensor, objects such as trees can have significant different motion patterns compared to its direct background. Dense grid methods can be helpful in such applications, or otherwise one should attempt to model the 3D world.

To stabilize the imagery will be advantageous for observations form moving platforms or moving or vibrating sensors, as well as for fixed sensors that have turbulence over a long line of sight. In the latter case the images will be disturbed in resolution as well as in motion. Corrections in the stabilization will allow the operator to observe in a more comfortable way.

\subsection{Stitching}

To generate large overview with a camera with only a limited field of view (FOV) image stitching is essential. When the individual images are part of a panorama or a complete hemisphere, recorded in a sequence in time, image stitching provide the means to generate a single image containing the image information from the entire scene. Overlapping areas are used in SBME to determine the motion (Schutte et al. 2003 [2]). The resulting image will show the operator a view of the scene that can immediately show interesting object in the surroundings, hence enhancing the awareness of the observer. Figure 6 shows an example of a mosaic generated from an air-to-ground image sequence, which contains a road, houses, and trees.
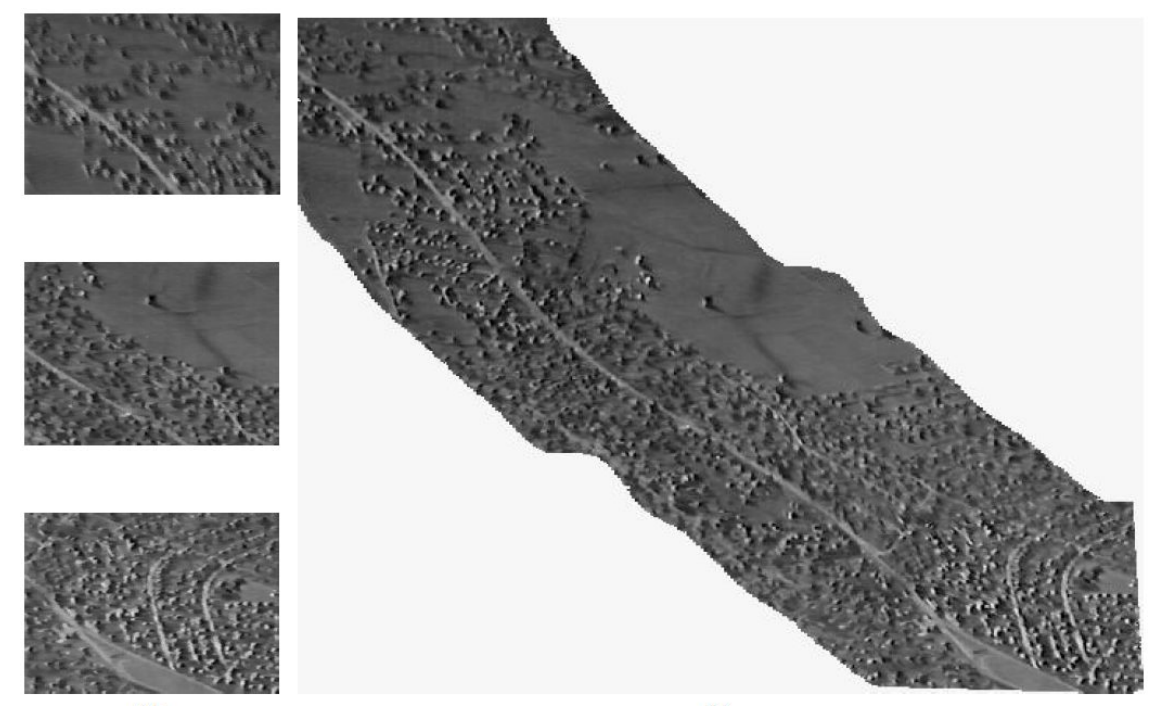

(a)

(b)

Figure 6. Example of mosaicking. (a) Sample images in the sequence. (b) Resulting mosaic. 


\subsection{Turbulence mitigation}

Atmospheric turbulence effects deteriorate imagery which is captured over long ground-to-ground ranges (van Eekeren et al. (1012)[6]). This diminishes the recognition range and it is therefore important to compensate visual degradation due to atmospheric turbulence. Turbulence compensation is a challenging problem because 1) the amount of turbulence is spatially varying due to anisoplanatic conditions while the isoplanatic angle, i.e. the angle over which the turbulence can be assumed constant, varies with atmospheric conditions and 2) the amount of turbulence varies significantly in time. Long range imagery over land and sea are limited by turbulence effects.

A hardware approach (Roddier, 1999) [7] is often used within astronomy applications. Except for space telescopes, all high-end telescopes are hindered by the Earth's atmosphere. Modern telescopes typically include an adaptive optics part, which corrects the atmospheric distortion. A typical assumption in such a system is that the turbulence effect is constant over the field of view of the telescope. For astronomy applications the imaging path is an almost vertical path through the atmosphere and effectively resulting in one or two phase screens. However, for ground-to-ground applications the full imaging path is a near horizontal path through the atmosphere, which has likely many variations of the refractive index. This will result in a more complex turbulence setup with multiple phase screens. In effect, the isoplanatic angle will often be much smaller than the field of view of the camera system, up to the level where it is even smaller than the IFOV. This means that a closed loop adaptive optics system, such as used in astronomy applications, is not suitable for ground-to-ground imaging.

The second approach is a software-based approach where the images are processed (preferably in real-time) after recording. There exist many different algorithms which can be divided into the following basic categories: global and local processing (Schwering et al., 2007[1]; van Eekeren et al., 2012[8]). Local processing can be done on a spatial and/or temporal level. In the global processing approach global registration and deconvolution is done and all frames are processed. Such an approach is useful for images captured under weak turbulence conditions. When the turbulence conditions are stronger (and the anisoplanatic angle is smaller) the variations within and between the frames are larger. This requires a local processing approach. A well-known local temporal approach is lucky-imaging. Here, the 'lucky' (best quality) frames are automatically selected for processing. While lucky imaging approaches can obtain very good results, its downside is the amount of time needed before a sufficient good image patch is seen for every image position.

In Figure 7 an example of urban turbulence compensation is presented. The recordings were made with the TNO SPITS system (Theil at al. (2006)[5]. The global approach is applied on these data, clearly resulting in improved image quality after processing. In the figure we see the recorded urban visible light image (left) and image processed with turbulence compensation (right).

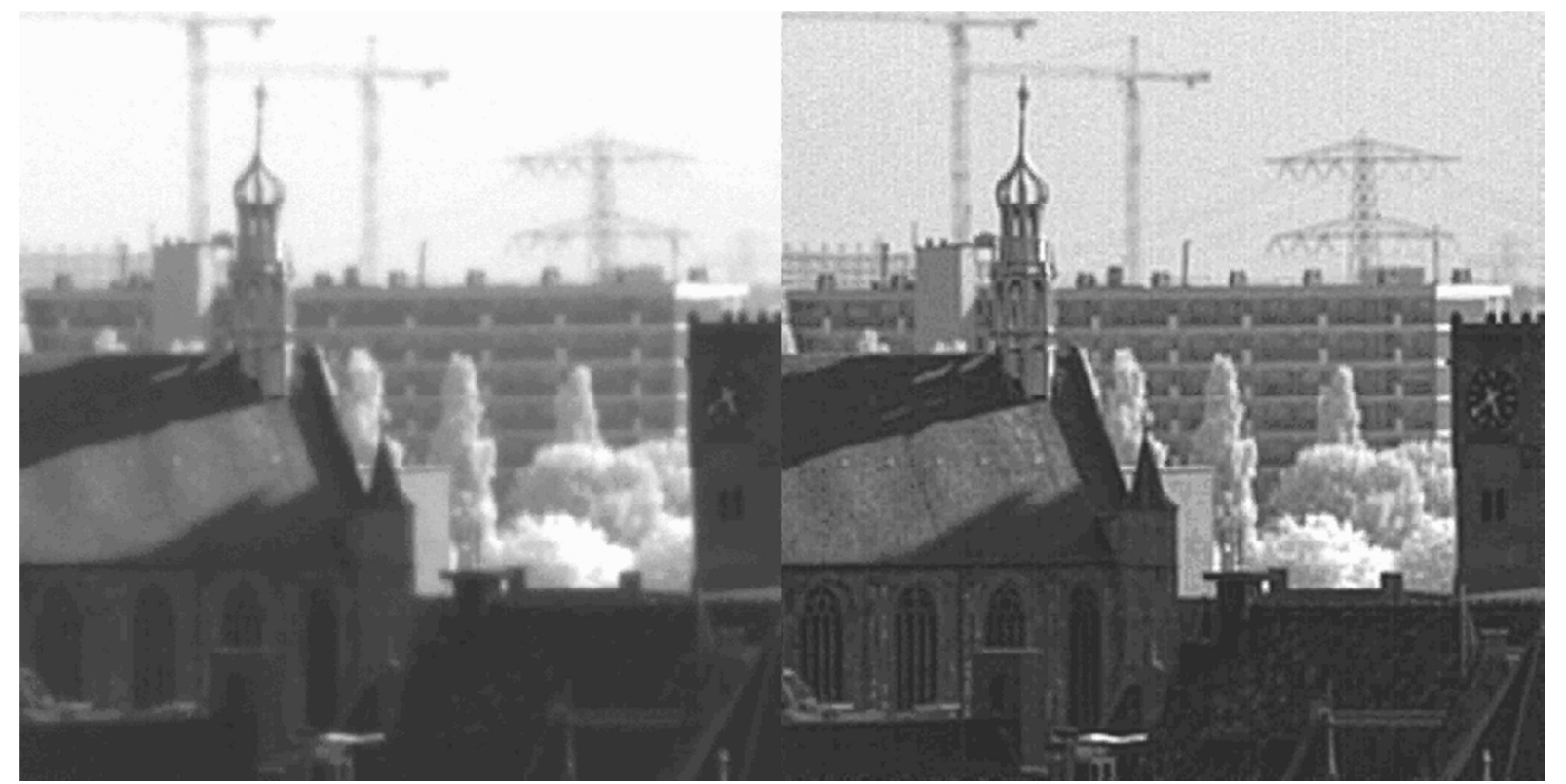

Figure 7. Originally recorded urban visible light image (left) and image processed with turbulence corrector (right). Images were recorded with the TNO SPITS system. 
Experiments were executed on the data gathered in the SET-165 trial held in Dayton Ohio in October 2011 (Velluet et al. 2012 [9]). In a two-step process image motion and image blurring are corrected. The obtained correction on the image sequence applied to these data is given in Figure 8. Detailed information, such as the letters and the checker board, is better visible in the corrected image in the figure.

Phase diversity (van Eekeren et al, 2011)[10] is a typical mixture approach in which dedicated hardware is used in combination with post-processing (software). Phase diversity does not record a single image, but several (often two) images simultaneously. These different images differ by a known phase aberration, such as a defocus. Having multiple images allows recovering its phase components. In turn, knowing its phase, allows to truly correct any distortion, where the other approaches typically resort to a phase-free blind deconvolution deblur approach. Similar to such deblurring approaches the phase diversity approach can be applied to non-isoplanatic imaging conditions. This approach aims to use, instead of 'basic' phase diversity, only one optical path containing a wavefront modulator which sweeps rapidly through the aberration configuration space. When its scan rate is higher than the rate of the atmospheric turbulence, this guarantees that 'lucky' images are seen. A disadvantage of this approach is that the modulations must be generated really fast and therefore expensive hardware (wavefront modulator) is needed.
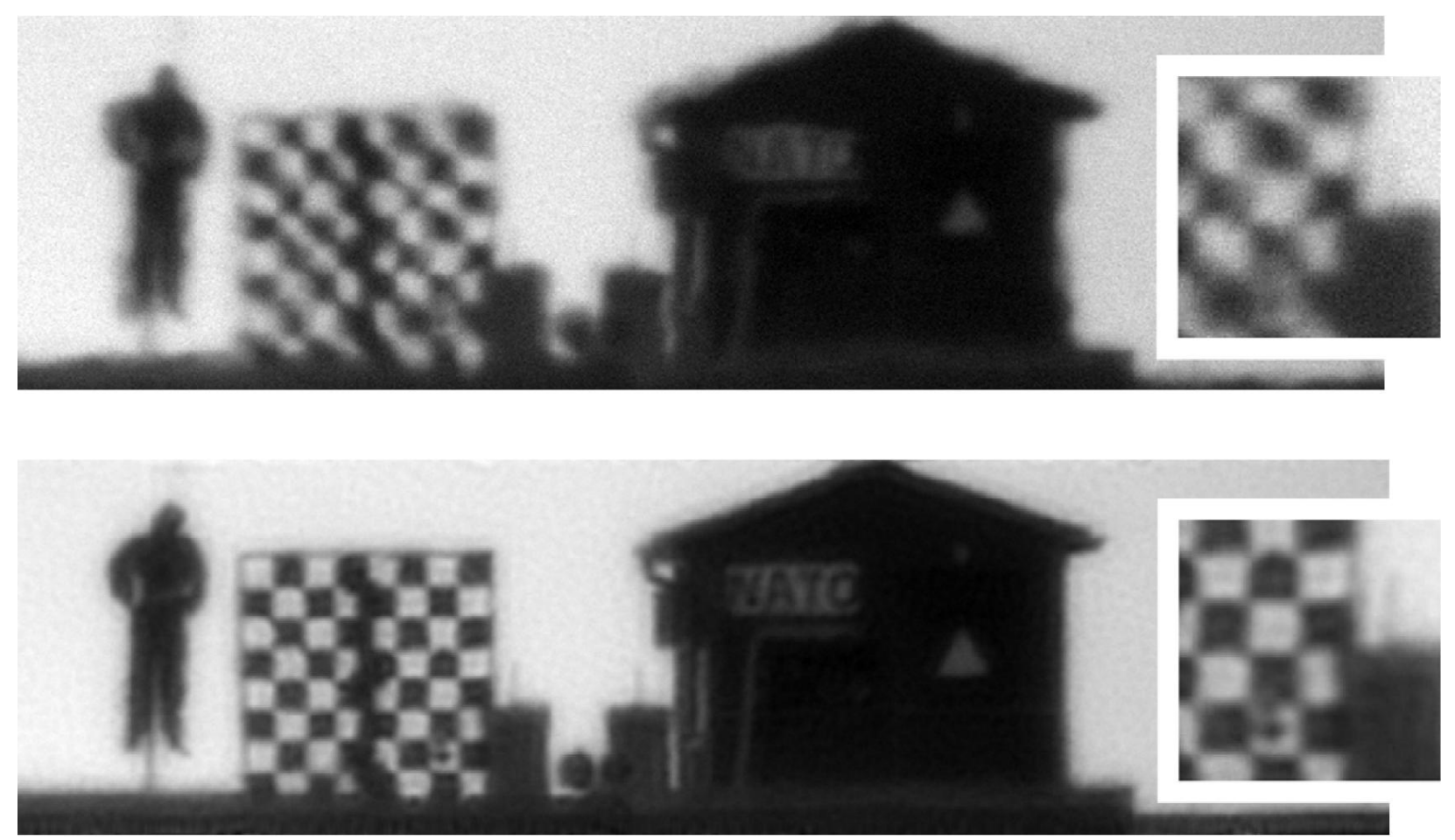

Figure 8. (top) One of the deteriorated frames of the SET-165 "NATO" sequence taken on 12 October 2011 at $15.35 \mathrm{~h}$ with on the right side a detail of the checkerboard. (bottom) Corresponding frame processed with the local-based method $\left(\mathrm{C}_{\mathrm{n}}^{2}=6.910^{-16} \mathrm{~m}^{-2 / 3}\right)$.

\subsection{Automated processing}

One implicit output of the DSR algorithm is a cue for scene change. Under the assumption of a slowly varying scene, only small scene changes per scene can be expected. However, in the presence of moving targets these scene changes will be significant at the locations of these targets. This allows these scene changes to be used as moving target indicators, which together with a proper tracking algorithm can be used for Moving Target Detection (MTD). Obviously, random motion patterns by moving objects cannot be modeled by the generic motion models used in SBME. Whenever moving objects are large and/or high-contrast, they will adversely contribute to SBME's accuracy. However, since moving object cues already are available by the DSR algorithm, identification of static scene elements is not difficult. Subsequently, the static scene elements can be used as SBME inputs, leading to an undisturbed motion estimate. 
Automated processing can first provide cues on targets and threats. The next step is to assess the activity pattern of the threat, which provide important information on the intent of the threat. In Bouma et al. (2013)[11] progress is shown on this matter.

For army applications several approaches for improvement of information are being followed. Above we have described image enhancement technology, we now describe automated detection and recognition processes. One study is from the operational perspective and forecasting the Electro-optical sensor and processing requirements for future urban operations (Schwering et al., 2013)[12]. From that study main results are that increase of quality of information from sensors and decrease in reaction time are obtained by advanced heterogeneous sensor fusion processes based on multi sources, and automated detection with robust clutter rejection and classification of intent. New imaging sensor technologies provide solutions for high spatial resolution imagery, such as those based on the above mentioned techniques.

One of the airborne sensors in the urban scenario will be a hyperspectral sensor. Different techniques are studied in a European context (DUCAS project). First study results are shown in Renhorn et al. (2012)[13] and more specific on change detection in Dekker et al. (2013)[14]. Combination of information from various sources and various processing techniques is the key parameter.

\section{TECHNOLOGY ASSESSMENT}

In this section we summarize the assessment of the different technologies. A first assessment is normally technical performance evaluation of the imagery, like resolution and signal-to-noise ratio. A second assessment requires the gain for the human task. Several experiments were carried out to address the operator gain. Target Acquisition (TA) range is one of the key performance parameters of a sensor system with the human-in-the-loop. Hence, the increase in TA range is a good measure of the effectiveness of signal processing techniques. For conventional scanning and staring systems it has been shown that the Triangle Orientation Discrimination (TOD) method is a good predictor of TA performance. In this section we investigate the applicability of the TOD to quantify the effectiveness of signal processing.

In their work Bijl et al. (2006) [17] Beintema et al.(2008)[16] Dijk et al (2013) [18] presented the effectiveness of signal processing techniques while performing operational tasks. By using the TOD approach (Triangle Orientation Discrimination) in experiments with human operators they showed significant improvements in task performance by applying techniques as LACE and DSR in different situations.

Assessment of the results of turbulence compensation is described in van Eekeren et al. (2013)[15]. They propose an image quality metric and good results are obtained. The results show qualitatively and quantitatively that the proposed method is capable of stabilizing and deblurring such image sequences. Analysis of 10 processed sequences show a blur reduction up to a factor of 2.1. Furthermore the scene is stabilized with a resulting tilt smaller than 0.5 pixel. These results enable an operator to see more details at large distances.

Recent developments take place to implement this technology for army application. With Photonis work is performed to add image enhancement to digital image intensifiers. With Adimec work is performed to add image enhancement to fullHD visual sensors.

\section{CONCLUSIONS}

We have shown different image enhancement techniques that are studied in the army context. We have shown the advantages of application of these techniques by use of different examples, and we have also addressed their quantitative performance assessment. The techniques provide:

- Better observation over long range

- Better observation in foggy and rainy conditions

- Cost effective digital zoom in IR imaging

- More effective use by operator by automatic detection and classification of specific objects

- No operator efforts are required in setting camera/monitor settings 


\section{ACKNOWLEDGEMENTS}

The authors would like to thank all participants in the various projects in the Electronic Defence and Intelligent Imaging departments at TNO that led to the development of the image enhancement processing. Financial support from the Netherlands Ministry of Defence in various projects is acknowledged.

\section{REFERENCES}

[1] Schwering P.B.W., van den Broek S.P., van Iersel M., 2007, in 'Infrared Technology and Applications XXXIII', eds. B.F. Andresen, SPIE Vol. 6542-100, 654230, Orlando Florida (USA), April 9-13, 2007: "EO System Concepts in the Littoral"

[2] Schutte, K., de Lange, D.J.J., van den Broek, S.P., "Signal conditioning algorithms for enhanced tactical sensor imagery," SPIE Proc. Vol. 5076, 92-100, (2003)

[3] Judith Dijk, Richard J. M. den Hollander, John G. M. Schavemaker, Klamer Schutte, "Local adaptive contrast enhancement for color images," Proc. SPIE 6575, Visual Information Processing XVI, 65750A,doi:10.1117/12.716886, (2007)

[4] Kemp, R.A.W., de Groot, J.F., van den Broek, S.P., de Lange, D-J.J., Dijk, J., Schwering P.B.W., in SPIE Vol 6943-0Y, Orlando-FL (USA), April 2008, "Results of Optical Detection Trials in Harbour Environment"

[5] Theil, A., Huizing, A.G., van Heijningen, A.W.P., Single Picture Integration for Territorial Waters Surveillance (SPITS): An initiative to improve situational awareness in littoral waters, MAST (Maritime Systems and Technology) Conference, Nice France, 4-6 September 2006

[6] van Eekeren, A. W. M., Kruithof, M. C., Schutte, K., Dijk, J., van Iersel, M., and Schwering, P. B. W., "Turbulence compensation: an overview," Proc. SPIE Infrared Imaging Systems: design, analysis, modeling and testing XXIII 8355 (2012)

[7] Roddier, F., ed., [Adaptive Optics in Astronomy], Cambridge University Press (1999).

[8] van Eekeren, A. W. M., Kruithof, M. C., Schutte, K., Dijk, J., van Iersel, M., and Schwering, P. B. W., "Patchbased local turbulence compensation in anisoplanatic conditions," Proc. SPIE Infrared Imaging Systems: design, analysis, modeling and testing XXIII, SPIE Vol. 8355 (2012)

[9] M.-T. Velluet, Mikhail Vorontsov, Gabriele Marchi, Stephane Nicolas, Piet Schwering, Jim Riker, 2012, Proc. SPIE 8380, in Atmospheric Propagation IX, 838001 (May 1, 2012); doi:10.1117/12.979459, "Turbulence characterization and image processing data sets from a NATO RTO SET 165 trial in Dayton (OH)"

[10] Adam W. M. van Eekeren, Klamer Schutte, Judith Dijk and Piet B. W. Schwering, "Time-varying phase diversity turbulence compensation," Proc. SPIE 8012, 80120D (2011); doi:10.1117/12.884580

[11] Henri Bouma, Gertjan Burghouts, Leo de Penning, Patrick Hanckmann, Johan-Martijn ten Hove, Sanne Korzec, Maarten Kruithof, Sander Landsmeer, Coen van Leeuwen, Sebastiaan van den Broek, Arvid Halma, Richard den Hollander, Klamer Schutte, "Recognition and localization of relevant human behavior in videos," Proc. SPIE, vol. 8711, (2013).

[12] Schwering, P.B.W. Grönwall, C., Future Electro-Optical sensors and processing in Urban Operations, SPIE, Dresden, 2013

[13] Renhorn, I., Axelsson, M., Benoist, K., Borghys, D., Boucher, Y., Briottet, X., De Ceglie, S., Dekker, R., Dimmeler, A., Dost, R., Friman, O., Kåsen, I., Maerker, J., van Persie, M., Resta, S., Schwering, P., Shimoni, M. and Haavardsholm, T.V., "Detection in Urban scenario using Combined Airborne imaging Sensors," Proc. SPIE 8353 (2012).

[14] Rob J. Dekker, Piet B.W. Schwering, Koen W. Benoist, Stefano Pignatti, Federico Santini, Ola Friman, SPIE [8743-14] 2013, LWIR Hyperspectral Change Detection for Target Acquisition and Situation Awareness in Urban Areas 
[15] Adam W.M. van Eekeren, Klamer Schutte, Judith Dijk Piet B.W. Schwering, "Quantitative evaluation of turbulence compensation," Proc SPIE Vol. [8706-22], (2013)

[16] Beintema, J.A., Bijl, P., Hogervorst, M.A., Dijk, J. (2008). Target Acquisition performance: effects of target aspect angle, dynamic imaging and signal processing. In: Infrared Imaging Systems: Design, Analysis, Modeling, and Testing XIX, SPIE $6941169410 \mathrm{C}$.

[17]Bijl, P., Schutte, K., Hogervorst, M. A., “Applicability of TOD, MTDP, MRT and DMRT for dynamic image enhancement techniques, In Defense and Security Symposium (pp. 62070E-62070E). International Society for Optics and Photonics, (2006)

[18]Dijk, J., van Eekeren, A. W., Toet, A., den Hollander, R. J., Schutte, K., van Heijningen, A. W., Bijl, P., "Evaluation of intensified image enhancement through conspicuity and triangle orientation discrimination measures," Optical Engineering, 52(4), 041105-041105, (2013) 